\title{
Die EU-Asylpolitik als Folge der Etablierung einer gemeinsamen Außengrenze
}

Weil kontrollfreie Binnengrenzen Asylsuchenden zu ermöglichen schienen, sich relativ ungehindert im Schengen-Raum zu bewegen, waren schon Ende der 1980er Jahre Regelungen über den gemeinsamen Umgang mit Asylanträgen diskutiert worden. Beinahe zeitgleich mit dem Schengener Durchführungsübereinkommen, das bereits einige Asylbestimmungen enthält, wurde 1990 in Dublin das „Übereinkommen über die Bestimmung des zuständigen Staates für die Prüfung eines in einem Mitgliedstaat der Europäischen Gemeinschaften gestellten Asylantrages“ unterzeichnet. ${ }^{1}$ Es trat 1997 nach langwierigen Debatten über die Umsetzung in Kraft. Zum einen legt es fest, dass ein Asylverfahren in dem EU-Staat durchzuführen ist, in den die oder der Asylsuchende zuerst eingereist ist. Auf diese Weise soll erreicht werden, dass Asylsuchende nicht von einzelnen Staaten abgewiesen werden können und folglich ohne Verfahren im Schengen-Raum von Land zu Land reisen (diskutiert unter der Formel ,refugees in orbit“). Darüber hinaus soll die Regelung im Falle einer Ablehnung eines Asylantrags durch einen Mitgliedstaat verhindern, dass in einem anderen Land ein weiterer Antrag gestellt wird (das sogenannte „Asylshopping“) (Lavenex 2001).

Weil für die Aufnahme von Asylsuchenden und die Durchführung von Asylverfahren in erster Linie Staaten an den EU-Außengrenzen zuständig sind, führte das Dublin-System zu erheblichen Ungleichgewichten (in den 1990er Jahren vor allem zulasten der Länder mit einer Grenze zum europäischen Osten, seit Anfang der 2000er Jahre vor allem der Mittelmeeranrainerstaaten Spanien, Italien und Griechenland). Ein Mechanismus zur Weiterverteilung von Schutzsuchenden innerhalb der EU erwies sich aber wegen des Widerstands diverser Staaten, zuletzt insbesondere solche im Osten Europas, als nicht durchsetzbar. Auch die großen

\footnotetext{
${ }^{1}$ https://eur-lex.europa.eu/legal-content/EN/TXT/PDF/?uri=CELEX:41997A0819(01)\&fro $\mathrm{m}=\mathrm{DE}(31.08 .2020)$.
} 
Unterschiede zwischen den Asylsystemen der Mitgliedstaaten ließen sich nicht auflösen. Zwar vereinbarten die Mitgliedstaaten ein „Gemeinsames Europäisches Asylsystem“. Es vermochte aber nur im Ansatz zur Vereinheitlichung der nationalen Asylregelungen beizutragen. EU-Parlament und Europäische Kommission mahnten seit den 1990er Jahren meist eine engere Zusammenarbeit der Mitgliedstaaten an, führten die Vorteile der Einführung gemeinsamer Regeln an und zielten tendenziell auf eine Stärkung der Rechte von Schutzsuchenden. Der Europäische Rat als Organ der Vertretung der Mitgliedsländer nahm demgegenüber in der Regel eine deutlich restriktivere Position ein.

Auch zwischen den Mitgliedstaaten und in den jeweiligen innenpolitischen Debatten lassen sich weitreichende Auseinandersetzungen über die Ausrichtung der Asylpolitik ausmachen. Während einige Staaten Rechte von Schutzsuchenden gewahrt wissen wollen, verweisen andere auf einen Primat der Kontrolle und Begrenzung von Zuwanderung. Seit Langem werden Fragen nach der Teilhabe von Schutzsuchenden am Arbeitsmarkt ebenso kontrovers diskutiert wie die Gewährung von Sozialleistungen oder von Bewegungsfreiheit in den Mitgliedstaaten. An den Debatten nehmen in den EU-Ländern wegen der je verschiedenen Asylsysteme zudem Akteure auf unterschiedlichen Ebenen teil: Die Gewährung von Leistungen und Handlungsmöglichkeiten für Schutzsuchende sind in einigen Fällen ausschließlich gesamtstaatliche Aufgaben, in anderen wiederum werden Verantwortlichkeiten föderal geteilt oder gehören in den Kompetenzbereich der Kommunen.

Anfang der 2000er Jahre beschloss die EU Mindeststandards für die nationale Asylgesetzgebung und die Asylverfahren - weniger im Interesse von Schutzsuchenden, sondern vor allem um auszuschließen, dass Schutzsuchende angesichts sehr unterschiedlicher Standards weiterwandern, um bessere Bedingungen in anderen Ländern zu finden. Die Mindeststandards blieben allerdings durch nationale Ermessensspielräume beschränkt, weshalb die Unterschiede in den Verfahren und in den Leistungen, die Asylsuchenden und anerkannten Flüchtlingen gewährt werden, weiterhin ausgesprochen groß sind. Hinzu kommt, dass nicht in allen Mitgliedstaaten die Mindeststandards erreicht werden - nicht zuletzt, weil aus der Sicht vieler staatlicher Akteure lange Verfahren, geringe Leistungen und wenige Rechte dazu beitragen könnten, die Zahl der Asylanträge niedrig zu halten, seien doch so „Pull-Faktoren“ auszuschließen (Boswell und Geddes 2011, S. 150-175).

Vor diesem Hintergrund vermochten sich die Schengen- bzw. EU-Staaten im Jahrzehnt vor und nach der Jahrtausendwende vor allem auf Maßnahmen zu einigen, die auf eine Verstärkung von Grenzkontrollen und Grenzschutz sowie eine Verminderung der Zahl der Menschen ausgerichtet waren, die an den Außengrenzen Asyl beantragen konnten. An Bedeutung gewannen hierbei die Übernahme 
von Grenzkontrollaufgaben durch Drittstaaten und die Förderung von bzw. die Forderung nach Einführung von Pass-, Visa- und Grenzkontrollen im Vorfeld der EU-Grenzen. Im Sinne einer ,remote control“ (Aristide Zolberg) wurden nicht nur außerhalb der EU vermehrt Bewegungen von Menschen überwacht, sondern diese möglichst auch immobilisiert. Instrumente zur Realisierung von Vorverlagerungen von Grenzkontrollen bilden seit den 1990er Jahren vor allem Regelungen, andere Staaten zu „sicheren Drittstaaten“, „sicheren Herkunfts- und Transitstaaten“ zu erklären und sie damit für Asylverfahren verantwortlich zu machen (Geiger und Pécoud 2010).

Solche Regelungen sind häufig abgestützt worden durch Verträge mit Herkunfts- oder Transitstaaten, die keineswegs immer bereit und in der Lage waren, Menschenrechte zu garantieren. Kooperations- und Assoziierungsabkommen der EU mit Drittstaaten unter anderem im Rahmen der „Europäischen Nachbarschaftspolitik“ umfassen etwa als „Mobilitäts-“ oder „Migrationspartnerschaften" regelmäßig migrationspolitische Bestimmungen (Bendel und Ripoll Servent 2018). Schwerpunkte bildeten zunächst vor allem Aspekte des Grenzschutzes, der Kontrolle von Bewegungen und der Rückübernahme von Angehörigen der Drittstaaten. Bald wuchs zudem die Bedeutung der Vermittlung von Informationen über Bewegungen weit vor den EU-Außengrenzen und der Abstimmung über die Frage, auf welche Weise potenzielle Bewegungen verhindert werden könnten.

„Mobilitätspartnerschaften“ hat die EU bislang mit neun Staaten geschlossen: der Republik Moldau (2008), Cap Verde (2008), Georgien (2009), Armenien (2011), Aserbaidschan (2013), Marokko (2013), Tunesien (2014), Jordanien (2014) und Belarus (2016). Diese Staaten sollen unerwünschte Migration blockieren und sind zur Rücknahme jener Migrantinnen und Migranten verpflichtet, die keinen Aufenthaltsstatus in der EU haben oder erhalten. Anreize für den Vertragsabschluss bestehen in wirtschaftlicher und finanzieller Unterstützung sowie in der Liberalisierung der Visabestimmungen. Das Instrument der „Migrationspartnerschaften" trat als Reaktion auf den Anstieg der Zahl der Schutzsuchenden in der EU 2015/2016 hinzu: Vornehmlich mit nord-, west- und ostafrikanischen Staaten sollen sie abgeschlossen werden, um Bewegungen in Richtung Europa zu verhindern oder Menschen aus Europa unter erleichterten Bedingungen zurückführen zu können. Anders als im Falle der Mobilitätspartnerschaften sind Visaerleichterungen nicht vorgesehen, im Vordergrund stehen entwicklungs- und handelspolitische Kooperationen.

Wie ein Blick in die Asylstatistik der 2010er Jahre zeigt (Tab. 5.1), blieb die EU bzw. blieben einzelne Mitgliedstaaten trotz der geschilderten Maßnahmen zur 
Tab.5.1 Zahl Asylsuchender in den 28 Mitgliedstaaten der EU 2011 bis 2019

\begin{tabular}{l|l|l}
\hline Jahr & Zahl & davon Deutschland \\
\hline 2011 & 309.045 & 53.240 \\
\hline 2012 & 335.290 & 77.485 \\
\hline 2013 & 431.100 & 126.705 \\
\hline 2014 & 626.965 & 202.645 \\
\hline 2015 & 1.322 .850 & 476.510 \\
\hline 2016 & 1.260 .920 & 745.160 \\
\hline 2017 & 712.250 & 222.565 \\
\hline 2018 & 664.410 & 184.180 \\
\hline 2019 & 743.595 & 165.615 \\
\hline
\end{tabular}

Quelle: Eurostat

Verminderung der Asylzuwanderung dennoch wichtige Ankunfts- und Aufnahmestaaten der Asylmigration. Warum aber wurde Europa, und hier insbesondere Deutschland, Mitte der 2010er Jahre ein Hauptziel der Bewegung insbesondere syrischer Schutzsuchender? Sechs Elemente eines komplexen Zusammenhangs seien hier skizziert. Die Reihenfolge der Argumente repräsentiert keine Hierarchie, alle genannten Faktoren stehen in einem unmittelbaren Wechselverhältnis zueinander:

1. Finanzielle Mittel: Wichtige Herkunftsländer von Asylsuchenden in der EU lagen in relativer geografischer Nähe (Syrien, Südosteuropa). Die Kosten für das Unternehmen Flucht von dort hielten sich mithin in Grenzen - zumindest im Vergleich zu Bewegungen aus anderen globalen Konfliktherden etwa in West- oder Ostafrika, Südasien oder Lateinamerika, die in den vergangenen Jahren und Jahrzehnten selten Europa erreichten. Außerdem grenzt die Türkei als das wichtigste Erstziel des Großteils syrischer Schutzsuchender unmittelbar an EU-Länder - und schien zugleich vor dem Hintergrund der mehr als drei Millionen Schutzsuchenden im Land, eines prekären Aufenthaltsstatus und beschränkter Möglichkeiten des Zugangs zu Bildung und zum regulären Arbeitsmarkt nur geringe Zukunftsperspektiven zu bieten.

2. Netzwerke: Migration findet vornehmlich in Netzwerken statt, die durch Verwandtschaft und Bekanntschaft konstituiert sind. EU-Staaten und hier insbesondere Deutschland entwickelten sich Mitte der 2010er Jahre auch deshalb zu einem wichtigen Ziel von Asylsuchenden, weil es hier (mit allerdings erheblichen Unterschieden zwischen den EU-Staaten, siehe oben) zum Teil seit 
Längerem recht umfangreiche Herkunftskollektive gab, die für Menschen, die vor Krieg, Bürgerkrieg und Maßnahmen autoritärer Systeme auswichen, eine zentrale Anlaufstation bildeten. Das galt nicht nur für Menschen aus Syrien und Südosteuropa, sondern auch für solche aus dem Irak, Afghanistan und Eritrea. Und weil migrantische Netzwerke die Wahrscheinlichkeit für weitere Migration erhöhen, hat die Zuwanderung von Asylsuchenden die insbesondere in den Jahren 2014 bis 2016 zu beobachtende Dynamik gewonnen.

3. Aufnahmeperspektiven: Staaten entscheiden mit weiten Ermessensspielräumen über die Zulassung von Migration und den Status jener, die einen Schutzstatus erhalten. Die Bereitschaft, Schutz zu gewähren, ist immer ein Ergebnis vielschichtiger Prozesse des Aushandelns durch Individuen, Kollektive und (staatliche) Institutionen, deren Beziehungen, Interessen und Kategorisierungspraktiken sich stets wandeln. Mit der permanenten Veränderung der politischen, administrativen, publizistischen, wissenschaftlichen und öffentlichen Wahrnehmung von Migration verbindet sich ein Wandel im Blick auf die Frage, wer unter welchen Umständen als schutzsuchend verstanden und wem in welchem Ausmaß und mit welcher Dauer Schutz oder Asyl zugebilligt wird. In den frühen 2010er Jahren und bis weit in das Jahr 2015 hinein ließ sich, in höherem Maße als in vielen anderen EU-Staaten, eine relativ große Aufnahmebereitschaft in der Bundesrepublik Deutschland beobachten. Verantwortlich dafür war eine nicht zuletzt vor dem Hintergrund der günstigen Situation von Wirtschaft und Arbeitsmarkt positive Zukunftserwartung in Politik, Wirtschaft und Gesellschaft. Die seit vielen Jahren laufende breite Diskussion um den Fachkräftemangel und um den demografischen Wandel führte ebenso zu einer Öffnung wie die Akzeptanz menschenrechtlicher Standards und die Anerkennung des Erfordernisses des Schutzes vornehmlich von Menschen aus Syrien, aus der zugleich eine große Bereitschaft zu ehrenamtlichem Engagement resultierte.

4. Aufhebung von Migrationsbarrieren: Seit den 1990er Jahren hatte die EU, wie geschildert, ein System zur Abwehr von Fluchtbewegungen aufgebaut. Eine vielgestaltige europäische migrationspolitische Zusammenarbeit mit Staaten wie Libyen, Ägypten, Tunesien, Marokko, Albanien oder der Ukraine suchte zu verhindern, dass potenzielle Asylsuchende die Grenzen der EU erreichten (Geiger und Pécoud 2010; Walton-Roberts und Hennebry 2014; Gammeltoft-Hansen 2011). Diese EU-Vorfeldsicherung brach seit Anfang der 2010er Jahre aufgrund der Destabilisierung diverser dieser Kooperationspartner am Rand der EU (unter anderem im Kontext des „Arabischen Frühlings“, aber auch des Ukraine-Konflikts) zusammen. Der Zerfall der politischen Systeme war eng verbunden mit den tiefgreifenden Folgen der weltweiten Finanz- 
und Wirtschaftskrise seit 2008, die die gesellschaftlichen Konflikte in zahlreichen EU-Anrainerstaaten verschärfte, die staatlichen Handlungsmöglichkeiten beschnitt sowie die Bereitschaft und die Reichweite einer Zusammenarbeit mit der EU minimierte.

5. Auflösung des „Dublin-Systems“: Die Weltwirtschaftskrise wirkte nicht nur auf den äußeren Ring der Vorfeldsicherung gegen die Zuwanderung von Schutzsuchenden jenseits der Grenzen der EU, sondern auch in den inneren Ring hinein. Das seit den frühen 1990er Jahren entwickelte europäische „Dublin-System“ führte tendenziell zu einer Abschließung der EU-Kernstaaten und insbesondere Deutschlands gegen weltweite Fluchtbewegungen, indem es die Verantwortung für die Durchführung eines Asylverfahrens jenen europäischen Staaten überließ, in die Antragstellende zuerst eingereist waren (Lavenex 2001). Das konnten nur Staaten an der EU-Außengrenze sein. Lange schien das System zu funktionieren, insbesondere deshalb, weil die Zahl der Schutzsuchenden, die europäische Grenzen erreichten, seit Mitte der 1990er Jahre relativ niedrig lag. Aufgrund der Finanz- und Wirtschaftskrise und im Kontext des Anstiegs der Zahl der Asylsuchenden aber waren diverse europäische Grenzstaaten, vornehmlich Griechenland und Italien, immer weniger bereit und in der Lage, die ungleich verteilten Verantwortlichkeiten des DublinSystems zu tragen, die Schutzsuchenden zu registrieren und in das jeweilige nationale Asylverfahren zu fügen.

6. Die Bundesrepublik als „Ersatz-Zufluchtsland“: Die weltweite Finanz- und Wirtschaftskrise führte innerhalb der EU dazu, dass die Bereitschaft traditionsreicher und gewichtiger Asylländer wie etwa Frankreich oder Großbritannien erheblich sank, Schutz zu gewähren (siehe Punkt 3 zu den unterschiedlich gelagerten Aushandlungen über die Aufnahme von Schutzsuchenden in den EU-Staaten). In diesem Kontext wurde die Bundesrepublik 2015 gewissermaßen zu einem Ersatz-Zufluchtsland und damit zu einem neuen Ziel im globalen Fluchtgeschehen - neu insofern, als es bis dahin keine umfangreichen Bewegungen von Schutzsuchenden von außerhalb Europas in die Bundesrepublik gegeben hatte.

Mehrere wesentliche Faktoren trugen dazu bei, dass sich insbesondere seit Herbst 2015 die Konstellationen im Blick auf die Migration von Schutzsuchenden erneut weitreichend änderten: Von einem europäischen oder gar globalen Teilen von Verantwortung gegenüber den weltweiten Fluchtbewegungen ließ sich in kaum einer Hinsicht sprechen. Das Desinteresse bzw. die Weigerung vieler Staaten, Schutzsuchende aufzunehmen, mündete nicht nur allenthalben in Maßnahmen zur Abschließung von Grenzen, die dazu führten, dass Menschen auf der Flucht 
immobilisiert wurden oder neue Pfade einschlagen mussten, die mit größeren Risiken, höheren Kosten und geringeren Chancen, Schutz zu finden, verbunden waren. Ein Ergebnis des Scheiterns der seit Langem auch in der EU diskutierten Verantwortungsteilung bestand darin, dass Staaten bzw. deren Bevölkerungen, die zunächst noch in höherem Maße Bereitschaft zeigten, Schutzsuchende aufzunehmen, wie etwa die Türkei, der Libanon, Deutschland oder Schweden, relativ rasch ebenfalls auf einen politischen Kurs der Grenzschließung und des Desinteresses gegenüber einer Aufnahme einschwenkten. Allenthalben fielen nun auch hier Asylsuchende unter den Generalverdacht einer potenziellen Belastung und Bedrohung für Sicherheit, wirtschaftliche Prosperität, soziale Systeme oder spezifische kulturelle und politische Werte einer vielfach als homogen vorgestellten Gesellschaft.

Die Europäische Union hatte zwar, wie geschildert, seit Mitte der 1990er Jahre mit dem Dublin-Abkommen und den Reformen der Jahre 2004 bis 2006 Instrumente zur „Harmonisierung“ asylpolitischer Regelungen der Mitgliedstaaten und von Asylverfahren entwickelt. Der Rahmen aber blieb fragmentiert, gewissermaßen ein in den Anfängen steckengebliebenes Projekt (Bendel 2017), dessen Anwendung in der Konstellation von 2015/16 scheiterte. Fortan beschränkte sich die Kooperation der EU-Mitgliedstaaten erneut wesentlich auf die Entwicklung von restriktiven Regeln für eine gemeinsame Grenz- und Visapolitik sowie auf die Zusammenarbeit zur Begrenzung der Asylzuwanderung, wie sie etwa im Rahmen des Abkommens mit der Türkei im März 2016 realisiert werden konnte. Sie geht einher mit einer Renaissance der Vorfeldsicherung: Stärker noch als im Jahrzehnt vor und nach der Jahrtausendwende sollen Verträge mit Staaten jenseits der Grenzen der Europäischen Union (potenzielle) Schutzsuchende bereits weit vor den EU-Außengrenzen aufhalten. Hinzu treten asylpolitische Verschärfungen im Innern der Mitgliedstaaten, die einen Beitrag dazu leisten sollen, (potenzielle) Schutzsuchende davon abzuhalten, den Weg nach Europa einzuschlagen.

Parallel dazu schien vielen Mitgliedstaaten eine verstärkte Grenzsicherung innerhalb des Schengen-Raums nötig zu sein. Mit dem Anstieg der Zahl der Asylsuchenden Mitte der 2010er Jahre verschärften sich Forderungen, die Hürden für die Durchführung von Grenzkontrollen im Schengen-Raum zu senken. Vermehrt sind solche seither wieder eingeführt worden. Weiterhin nehmen sechs der insgesamt 26 Schengen-Staaten an Teilen ihrer Staatsgrenzen - oder im Falle Frankreichs: innerhalb des eigenen Staatsgebiets - Personenkontrollen vor. Die Begründungen für die Wiedereinführung von Grenzkontrollen lauten bei allen Staaten ähnlich: Schutz vor globaler terroristischer Bedrohung, grenzüberschreitender organisierter Kriminalität und sogenannter ,secondary movements“ 
von Asylsuchenden innerhalb des Schengen-Raums ${ }^{2}$, also als Herausforderungen der Globalisierung markierte Phänomene, denen nicht allein durch europäische Zusammenarbeit, sondern auch durch die Betonung nationalstaatlicher Souveränität und des Schutzes der nationalen Territorien beizukommen sei.

${ }^{2}$ Europäische Kommission, Temporary Reintroduction of Border Control, 2019, https://ec. europa.eu/home-affairs/what-we-do/policies/borders-and-visas/schengen/reintroduction-bor der-control_en (20.09.2020). 
Open Access Dieses Kapitel wird unter der Creative Commons Namensnennung 4.0 International Lizenz (http://creativecommons.org/licenses/by/4.0/deed.de) veröffentlicht, welche die Nutzung, Vervielfältigung, Bearbeitung, Verbreitung und Wiedergabe in jeglichem Medium und Format erlaubt, sofern Sie den/die ursprünglichen Autor(en) und die Quelle ordnungsgemäß nennen, einen Link zur Creative Commons Lizenz beifügen und angeben, ob Änderungen vorgenommen wurden.

Die in diesem Kapitel enthaltenen Bilder und sonstiges Drittmaterial unterliegen ebenfalls der genannten Creative Commons Lizenz, sofern sich aus der Abbildungslegende nichts anderes ergibt. Sofern das betreffende Material nicht unter der genannten Creative Commons Lizenz steht und die betreffende Handlung nicht nach gesetzlichen Vorschriften erlaubt ist, ist für die oben aufgeführten Weiterverwendungen des Materials die Einwilligung des jeweiligen Rechteinhabers einzuholen. 